\title{
Análisis estratégico de la gerencia de investigación y desarrollo en los parques tecnológicos
}

\author{
Chirinos R., Ángel Rafael* \\ Rincón, Sorayda**
}

\section{Resumen}

El objetivo de la presente investigación es realizar un análisis estratégico de la gerencia de l+ D en los Parques Tecnológicos del Occidente del País. Se trata de un estudio de tipo descriptivo, no experimental para el cual se seleccionaron dos poblaciones: la primera, conformada por los Directores Ejecutivos de el Parque Tecnológico de Mérida (CITEC), el Parque Tecnológico de Barquisimeto (TECNOPARQUE) y el Parque Tecnológico Universitario del Zulia (PTU) y la segunda integrada por trece investigadores de los tres Parques tecnológicos ya nombrados; a estas poblaciones se les aplicó dos cuestionarios como instrumentos de recolección de información. Los resultados fueron procesados y analizados estadísticamente; reflejando que existen fortalezas en la estructura organizativa, disponibilidad de personal técnico gerencial, presencia de personal técnico calificado, ambiente organizacional favorable para la investigación. No obstante se destacan como aspectos limitantes, los pocos mecanismos de promoción y publicidad, bajo presupuesto asignado a los proyectos de investigación. En el análisis del posicionamiento externo se señalan como oportunidades el apoyo institucional y financiero que prestan instituciones conexas como FONACYT, la imagen institucional que representa el entorno universitario, el amplio mercado cautivo de la pequeña y mediana empresa y la amplitud de comunicación y vinculación con la sociedad civil, pero también se observan amenazas por la poca voluntad de la Banca Comercial Privada a financiar actividades de innovación, los altos costos de insumos-equipos, el escaso apoyo bibliográfico, la actual normativa universitaria y la resistencia del empresariado a establecer relaciones comerciales con las dependencias universitarias.

Palabras clave: Parques Tecnológicos, Gerencia Estratégica, Investigación y Desarrollo.

\section{Recibido: 06-08-31. Aceptado: 06-10-31}

* Profesor Titular de la Facultad de Ciencias Veterinarias de la Universidad del Zulia. Magister en Gerencia de Proyectos de Investigación y Desarrollo de la Universidad Rafael Belloso Chacín. Apartado 15252.

** Sociólogo, LUZ. Magíster en Planificación y Gerencia de C y T, LUZ. Doctora en Ciencias Gerenciales, Universidad Rafael Belloso Chacín. Docente de la Escuela de Sociología de LUZ. Investigadora del Centro de Estudios de la Empresa de la FCES de LUZ. Acreditada en el Programa de Promoción al Investigador del CONICIT. E-mail: sorarincon@hotmail.com 


\title{
Strategic Analysis of Research and Development Management in University Technological Parks
}

\begin{abstract}
The objective of this research was to undertake a strategic analysis of $R$ and $D$ management in technological parks in the western part of the country (Venezuela). It is a non-experimental descriptive study for which two populations were selected: the first made up of executive directors of the Mérida technological park (CITEC), the Barquisimeto Technological Park (Technoparque), and the University Technological Park in Maracaibo (PTU), and the second study population was thirteen researchers in the aforementioned parks. Questionnaires were applied to these populations as an information collection instrument. The results were processed statistically, giving evidence to the existence of strengths in the organizational structures, availability of technical management personnel, qualified technical personnel, and favorable environmental organization for research. However, limiting factors were inadequate promotional mechanisms, little publicity, and low operating budgets assigned to research projects. The analysis of external positioning indicated that: opportunities for institutional support and financing by institutions such as FONACYT existed, and the institutional image represented by the university, the ample market of small and medium sized industry and ample communication and relations with society were favorable. But there were threats due to little confidence on the part of the commercial banking sector to finance innovation as well as the high cost of equipment and supplies, little bibliographical support, inadequate university norms in relation to the same, and the resistance of private industry to establish commercial relations with the university.
\end{abstract}

Key words: Technological parks, strategic management, research and development.

\section{Introducción}

El proceso de transformación que vive el mundo entero en la actualidad, ha traído consigo el fortalecimiento de viejos modelos socioeconómicos de desarrollo. Venezuela no ha escapado a estas tendencias y viene experimentando, desde hace varios años, un proceso de cambio estructural.

Se ha oscilado desde un extremo del abanico de opciones hasta el otro, sin que mediara un proceso paralelo de capacitación y preparación efectiva del universo de los actores fundamentales del proceso. Dentro de este universo se incluyen grupos o instituciones como: el empresario nacional, los dirigentes de or- ganizaciones del sector estatal, la dirigencia de las instituciones nacionales de investigación y desarrollo, los consultores que demandan, como requerimiento fundamental para abordar el proceso de modernización y apertura comercial, un conjunto idóneo y actualizado de elementos como los siguientes: recursos humanos, información comercial y tecnológica, asistencia técnica, financiamiento, infraestructura legal, normas y estándares técnicos (Parisca, 1992).

Señala Viana et al, (1994) que Venezuela al igual que la mayoría de los países latinoamericanos, intenta desde el año 1989 desenvolverse según otro modelo económico, como respuesta a los requerimientos que se desprende de los 
nuevos giros que da la economía mundial y a los problemas que confronta su propia economía, esto implica una transición que va desde el agotamiento del modelo rentista, posible gracias al petróleo, hacia una economía productiva delineada según los cánones del mercado en un contexto de apertura comercial. Dicho intento ocurre en medio de ondas transformaciones tecnológicas que emplea nuevas e importantes exigencias para que tal modelo sea exitoso. Por otro lado, las nuevas tecnologías vienen acompañadas de un modelo organizativo y gerencial basado en el concepto de máxima flexibilidad y agilidad de respuesta, mínimo inventario y cero defectos, cuya expresión organizativa e institucional más acabada es la japonesa.

Baena (2003), afirma que la gran incidencia positiva que las actividades de I + D tiene sobre el desarrollo de los países ha conducido a los gobiernos de los diferentes estados a destinar una parte de sus recursos financieros a potenciar ambas actividades. De esta manera ha sido posible diversificar las líneas de investigación con la finalidad de abarcar cada vez más campos y al mismo tiempo, asegurar la formación de personal calificado. El desarrollo tecnológico consiste en trabajos sistemáticos basados en conocimientos ya existentes con el objetivo de fabricar nuevos productos, instaurar nuevos procesos, estadísticos, nuevos servicios o introducir mejoras sustanciales sobre los ya existentes.

Los países Latinoamericanos a la luz de la experiencia y avance logrado por los países desarrollados, también han implementado una variedad de modalidades de organización para propiciar la vincula- ción y gestionar la innovación y la transferencia de tecnología. Dada la importancia de la tecnología, las políticas se fundamentan en la identificación de los efectos negativos relacionados con la adquisición de tecnologías foráneas, además de propiciar las relaciones de las industrias con los centros de I + D que suelen ser precarias y sólo excepcionalmente cuentan con los laboratorios propios (Avalos, 1994).

Por su parte, Seatón (2000), señala que la misión de la mayoría de las organizaciones de investigación y desarrollo es contribuir, a través de la utilización de sus recursos técnicos y humanos a la prestación de servicios tecnológicos relacionados con la ingeniería, el desarrollo de productos y procesos, la investigación aplicada y los ensayos de laboratorio, a los procesos de asimilación, adaptación, generación y transferencia de tecnología que realizan los sectores productores de bienes y servicios dentro del entorno social.

Los Parques Tecnológicos se definen como un espacio físico que cuenta con infraestructura adecuada para la instalación de empresas productivas en los cuales, las actividades de investigación y desarrollo ocupan un lugar relevante en su operación, por tanto, estas unidades requieren de facilidades para la consecución de insumos tecnológicos, tales como recursos humanos de alto nivel, uso de infraestructura en centros de investigación, acceso a bibliotecas y servicios de documentación especializados, entre otros.

Las bondades de la creación de parques tecnológicos en otros países ha influido favorablemente para que FONACYT en abril de 1988 fomentara estudios de factibilidad en diversos estados 
del país, resultando tres modalidades de parques; incubadora de empresas o fabricas de fabricas, en la Universidad de Mérida; empresas de base tecnológica y de servicio de apoyo, en la Universidad Simón Bolívar y el centro industrial, en la ciudad de Barquisimeto, posteriormente en 1993 se crea el Parque Tecnológico Universitario (P.T.U) de la Universidad del Zulia.

Sin embargo, a pesar de las fortalezas que presenta los P. T. U, existen elementos que afectan el funcionamiento de estos y que de cierta manera impiden que los resultados de la vinculación de las Universidades con las empresas y sectores productivos se realice de manera satisfactoria, se supone que las causas de esta situación sean, entre otras, la limitación financiera por parte del Estado Venezolano y de parte de la Universidad, debilidades intra-universitaria, recursos administrativos y obstáculos administrativos, además el investigador no siente que es apreciada la importancia estratégica de su labor, el nivel de ingresos es muy bajo, al compararlos con sus homólogos en el exterior y por último cierta "rigidez" estructural que dificulta la identificación de oportunidades económicas y la explotación de los mismos.

El objetivo principal de esta investigación fue realizar un análisis estratégico de la gerencia de I+ D en los Parques Tecnológicos ubicados en el Occidente del País; identificar las fortalezas y oportunidades que afectan el funcionamiento de dichos Parques así como sus debilidades y amenazas.

La presente investigación se clasifica según el método utilizado como descriptiva no experimental y la población es- tuvo constituida por los tres Directores Ejecutivos de los Parques Tecnológicos de la región Centro Occidental y trece investigadores pertenecientes a estos Parques Tecnológicos.

El proceso de recolección de datos se realizó utilizando dos cuestionarios los cuales, una vez diseñados, fueron sometidos al proceso de validez y confiabilidad.

\section{Sobre la gerencia estratégica y la gerencia de investigación y desarrollo}

\subsection{Gerencia estratégica}

Se puede definir como la formulación, ejecución y evaluación de acciones que permitirán que una organización logre sus objetivos. La formulación de estrategias incluye la identificación de las debilidades y fortalezas de la organización, la determinación de las amenazas y oportunidades de ésta, el establecimiento de la misión, la fijación de objetivos y el desarrollo de estrategias alternativas.

La gerencia estratégica es la función de un equipo debidamente estructurado para actuar con sentido de proyección hacia el futuro, donde el gerente cumple el papel específico del estratega de la organización.

Se entiende por gerencia estratégica al proceso de seleccionar políticas y estrategias de acción administrativa, que permitan maximizar los resultados mediante el uso más adecuado de los recursos organizacionales, el cual se oriente tanto al análisis del contexto interno y externo como el pronóstico de las actividades futuras de la organización, incluyendo el desarrollo de los cuadros gerenciales (Villegas, 1991). 
En las empresas el proceso de la gerencia estratégica se desarrolla en tres niveles: el nivel estratégico de la gerencia, que se relaciona con la identificación de la misión de la empresa y con la selección de planes para lograr objetivos en cada una de las áreas de interés de la misma. Todo lo cual lleva al gerente a comprender, con mayor realidad, la imagen exacta de su papel, reconociendo la importancia de administrar los recursos técnicos, humanos y financieros de acuerdo a lineamientos estratégicos.

El nivel coordinativo, por su parte, se relaciona con los procesos de toma de decisiones que influencian las comunicaciones entre las unidades funcionales $u$ otras áreas de la organización. Tienen más que ver sobre la aplicación de estrategias de corto y mediano alcance y sirve de enlace entre las concepciones estratégicas y las operaciones o tácticas.

El nivel operativo de la gerencia estratégica se relaciona con los procesos de producción, mercadeo de bienes y servicios, mantenimiento y apoyo funcional para el movimiento diario de las actividades organizacionales.

El proceso de gestión dirigido al logro del funcionamiento eficiente de la empresa, mediante la aplicación sistemática de los objetivos estratégicos, planes y acciones, denominado gerencia estratégica, abarca la estructura organizacional de la empresa.

Según David (1990) la gerencia estratégica no significa tratar de predecir el ambiente, pues ello sería imposible en un medio como el nuestro. Se trata de realizar un análisis formal del entorno para definir escenarios, formular colegiadamente líneas de acción para cada uno, y tomar previsiones para una adecuada ejecución. La incertidumbre no constituye pretexto para no planificar estratégicamente, más bien la justifica.

\subsection{Elementos básicos de la gerencia estratégica}

Según David (1990), los elementos básicos de la gerencia estratégica son:

a) Los Estrategas: son individuos responsables del éxito o fracaso de una empresa. Ellos tienen diferentes títulos como ejecutivo, jefe, presidente, propietario, presidente de la junta, director ejecutivo, decano y empresario.

Debido a que los estrategas son seres humanos, ellos difieren en sus actitudes, valores, sentido de ética, deseo de asumir riesgos, preocupaciones de responsabilidad social, preocupación por la rentabilidad, preocupación por el corto plazo contra preocupación por el largo plazo, y estilo gerencial. Tienen también diferencias en cuanto a sus actitudes con respecto a la ética de las empresas.

b) La formulación de la misión: es lo que distingue a una empresa de otras parecidas, identifica el alcance de las operaciones de una empresa en los aspectos del producto y del mercado. Una formulación de misión incorpora la filosofía de los estrategas de una organización. Revela el concepto de una organización, su principal producto o servicio y las necesidades principales del cliente que la firma se propone satisfacer. Resumiendo, una formulación de misión clara y significativa describe los valores y prioridades de una organización. 
c) Las fortalezas internas: se refiere a actividades internas de una organización que se llevan a cabo especialmente bien. Las funciones de gerencia, mercadeo, finanzas, producción, investigación y desarrollo de un negocio deben auditarse o examinarse con el objeto de identificar y evaluar fortalezas internas de especial importancia. Las empresas exitosas siguen estrategias que las ayudan a beneficiarse de sus fortalezas internas, las empresas tanto sin ánimo de lucro como los comerciales, buscan al momento sacar provecho de sus fortalezas internas, estableciendo un enfoque de gerencia estratégica para la toma de decisiones.

d) Debilidades internas: es un término que se refiere a actividades de gerencia, mercadeo, finanzas, producción, investigación y desarrollo que limitan o inhiben el éxito general de una organización. Una firma debe tratar de seguir estrategias que efectivamente mejoren las áreas con debilidades internas.

e) Oportunidades externas: se refiere este término a las tendencias económicas, sociales, políticas, tecnológicas, competitivas, así como a luchas que podrían de forma significativa beneficiar a una organización en el futuro. En todos los frentes de nuestra sociedad ocurren ahora cambios masivos. La revolución de los computadores, la biotecnología, los cambios en la población, los cambiantes valores y actitudes con respecto al trabajo, la tecnología espacial, así como la cada vez mayor competencia de las empresas extranjeras son algunos de los cambios más importantes. Dichos cambios crean un tipo diferente de consumidor y como consecuencia, servicios y estrategias. Además de las tendencias am- bientales, las oportunidades externas inducen luchas que suceden una vez, tales como la aprobación de una ley, la decisión sobre un nuevo producto realizada por un competidor u algún adelanto tecnológico.

f) Las amenazas externas: es un término totalmente opuesto al anterior. Consisten ellas en tendencias económicas, sociales, políticas, tecnológicas y competitivas, así como hechos que son potencialmente dañinos para la posición competitiva presente o futura de una organización.

g) Los objetivos: son los resultados que una organización aspira a lograr a través de su misión básica. Los vocablos objetivos y metas se usan de diferentes formas en la literatura gerencial. Algunos autores los usan como sinónimos, otros utilizan el término "objetivos" para señalar resultados a corto plazo, mientras que "metas" se usa para referirse a logros a largo plazo.

Los objetivos son de vital importancia en el éxito de las organizaciones ya que suministran, dirección, ayuda en evaluación, crean sinergia, revelan prioridades, coordinación y son esenciales para las actividades de control, motivación, orden, y planificación efectivas. Deben reunir las siguientes características: ser medibles, claros, coherentes y estimulantes. En un conglomerado diversificado, los objetivos deben fijarse tanto para la empresa en general como para cada división.

h) Las estrategias: son los medios por los cuales se lograrán los objetivos. Las diversas estrategias empresariales incluyen expansión geográfica, diversificación, adquisición de competidores, obtención de control sobre proveedores o distri- 
buidores, desarrollando productos, penetración en el mercado, posicionamiento, liquidación, "asociaciones" o una combinación de algunos de estas acciones.

i) Metas: definimos la palabra "metas" como puntos de referencia para las aspiraciones que las organizaciones deben lograr, con el objeto de alcanzar en el futuro objetivos a un plazo más largo. Ellas deben ser medibles, estimulantes, coherentes y prioritarias.

Deben ser fijadas a niveles empresariales, divisionales y funcionales en una organización grande. Las metas deben formularse en términos de logro de gerencia, mercadeo, finanzas, producción e investigación y desarrollo. Ellas son especialmente importantes con la ejecución de estrategias, mientras que los objetivos son especialmente importantes para su formulación. Las metas representan la base para la asignación de recursos.

j) Políticas: se puede definir como la forma por medio de la cual las metas fijadas van a lograrse, o las pautas establecidas para respaldar esfuerzos con el objeto de lograr las metas ya definidas.

Hay dos características distintivas de las políticas: 1) son guías para la toma de decisiones; 2) Se establecen para situaciones repetitivas o recurrentes en la vida de una estrategia. Las políticas con frecuencia se formulan en términos de actividades de gerencia, mercadeo, finanzas, producción, investigación y desarrollo. Las políticas se pueden fijar a nivel empresarial y aplicarse en toda la organización o se pueden fijar a nivel funcional y aplicarse solamente a ciertos departamentos 0 actividades operativas.
Las políticas así como las metas son especialmente importantes en el proceso de ejecución de estrategias, pues ellas dan las líneas generales sobre las expectativas de organización con respecto a sus empleados y permitan coherencia y coordinación dentro de sus departamentos.

\subsection{El modelo de la gerencia estratégica}

El mismo autor refiere que el modelo de la gerencia estratégica abarca toda la empresa; va más allá de las operaciones, problemas y crisis cotidianas centrándose en el crecimiento y desarrollo globales de la organización. La estrategia se preocupa por el bosque, no por los árboles. La toma de decisiones estratégicas efectivas es la responsabilidad principal del propietario de una empresa o del ejecutivo. Las decisiones estratégicas incluyen establecer los negocios a que se va a dedicar la empresa, los negocios que se deben abandonar, la forma de asignar recursos, si es necesario ampliar operaciones o diversificar, la entrada en otros mercados geográficos, y si es o no necesario formar una empresa conjunta o fusionarse con otra firma.

El modelo global de dicho proceso de gerencia estratégica se puede resumir en doce pasos: establecer los objetivos, estrategias y la misión actual; realizar investigación externa con el objeto de identificar amenazas y oportunidades ambientales; realizar investigación interna con el objeto de identificar fortalezas y debilidades de la empresa; fijar la misión de la empresa; llevar a cabo análisis de for- 
mulación de estrategias con el objeto de generar alternativas factibles; fijar objetivos; fijar estrategias; fijar metas; fijar políticas; asignar recursos; analizar bases internas y externas para estrategias actuales; medir los resultados y tomar las medidas correctivas del caso.

El proceso de gerencia estratégica permite que una organización utilice efectivamente sus fortalezas con el objeto de aprovecharse de las oportunidades externas y reducir a un mínimo el impacto de las amenazas externas. Las actividades de formulación, de ejecución y evaluación de estrategias hacen posible que una organización desarrolle estrategias tanto ofensivas como defensivas. Nótese que el proceso de gerencia estratégica es a la vez dinámico y continúo. Un cambio en cualquiera de los componentes esenciales del modelo puede requerir una variación en uno o todos los demás componentes en cualquier punto del proceso. Ejemplo: un cambio económico puede representar una oportunidad o una amenaza externa importante y requerir una variación en las estrategias y objetivos de la compañía.

El hecho de no alcanzar metas funcionales o de división específicos puede requerir un cambio en los objetivos o políticas de la compañía; un competidor importante puede anunciar un cambio de estrategias que presenta una amenaza u oportunidad significativa. Los esfuerzos de ejecución, así como también los factores internos y externos, deben por tanto evaluarse de forma continua. El proceso de gerencia estratégica en realidad nunca termina. A veces se le denomina "proceso de gerencia de cambio estratégico" (David, 1990).

\subsection{Investigación y Desarrollo}

Los procesos de investigación y desarrollo (l y D), son considerados como uno de los puntos clave para el desarrollo del país; su objetivo principal es la búsqueda y producción del conocimiento a través de la investigación científica y tecnológica para lograr la innovación que busca contribuir a que exista una mejor calidad de vida, que es la meta del hombre actual.

Según Atul, citado por Figueroa (1996) la investigación y desarrollo es el proceso de producción de conocimientos científicos y tecnológicos. Se entiende por "investigación" el componente más fundamental y teórico de este proceso, en cambio el "desarrollo" consiste en usar el conocimiento básico generado mediante la investigación y en ponerlo en condiciones de ser aplicado.

Esta búsqueda de conocimiento, tanto científico como tecnológico, no la realiza el hombre de manera arbitraria, por lo que trata de que el proceso de investigación sea coherente, sistemático y bien coordinado para ofrecer resultados lo más precisos posibles. De esta idea surgen los centro o unidades de investigación, los cuales se definen como organizaciones donde un conjunto de personas llamadas grupos o equipos bien organizados, sujetos a ciertas reglas de operación y ligados a un mismo órgano de dirección, tratan de lograr una aproximación sistemática y ordenada de los nuevos conocimientos y principios universales y de preparar su aplicación o uso en el campo productivo o social. Estos conocimientos pueden ser generados a través de diferentes tipos de investigación, ya 
sean investigación aplicada, básica fundamental y básica aplicada.

Freeman citado por Figueroa (1996) distingue las siguientes categorías de investigación y desarrollo que son:

La Investigación Básica, es la investigación emprendida para conseguir nuevos conocimientos y comprensión científica, no esta dirigida primordialmente a ninguna meta o aplicación práctica específica, genera nuevas hipótesis, teorías y leyes, no complementa ninguna aplicación práctica, específica o inmediata pero se puede orientar hacia un área de interés para la entidad que la realiza.

Esta a su vez, se divide en (Figueroa, 1996): a) Investigación fundamental pura: el interés científico del investigador es el que determina el tema estudiado, se realiza generalmente en la universidades, en organizaciones sin fines de lucro o en laboratorio oficiales. b) Investigación fundamental orientada: la organización del investigador dirige normalmente su trabajo hacia un campo de interés científico, económico o social. c) Investigación aplicada: es también considerada investigación original comprendida para obtener nuevos conocimientos científicos o técnicos, pero se orienta hacia una meta u objetivo práctico específico. Se realiza para determinar posibles usos para los hallazgos de la investigación o para determinar nuevos métodos o modos de conseguir unos objetivos específicos y predeterminados. Los resultados de esta investigación son válidos para uno o para algunos productos, operaciones, métodos y sistemas; desarrolla ideas convirtiéndo- les en formas operacionales; el conocimiento o la información derivada de ella se puede patentar o no. d) Desarrollo Experimental: se emplea cuando el conocimiento científico es utilizado para producir materias primas, dispositivos, productos, procesos, sistemas o servicios nuevos o sustancialmente mejorados. El desarrollo experimental es el trabajo sistemático que se apoya en el conocimiento que se obtuvo de la investigación y/o de la experiencia práctica, dirigido a producir nuevas materias primas, productos y dispositivos, a instalar nuevos procesos $y$ sistemas y a mejorar los ya producidos e instalados. No toda la actividad de desarrollo es de carácter experimental, la experimentación es una característica dominante de esta fase de I y D.

\subsection{La Gerencia de investigación y desarrollo}

La gerencia es el arte y la ciencia de trabajar con y a través de un equipo de personas hacia el logro de los objetivos de una organización. Esto implica construir un cuerpo de conocimiento sobre dicha actividad, y que la actividad del gerente involucre relación con otras personas para lograr los objetivos de la organización (Padilla, 1990).

Las organizaciones dedicadas a la realización de actividades de I y $\mathrm{D}$ se deben administrar como cualquier empresa que produce bienes y servicios con la diferencia que estas organizaciones producen un producto: conocimiento.

Este conocimiento es para un propósito concreto. La I y $\mathrm{D}$ no produce un producto físico para la venta o un proceso operativo. No produce una nueva activi- 
dad, no produce calidad. Lo que produce es Know-how y el fundamento de otros resultados.

Este Know-how desarrollado por I y D, se traduce mediante una acción directiva, en procesos, productos, reducción de costos, mejoras de calidad, concordancia con la normativa de conservación del medio ambiente, apoyo a las demandas del producto y otros objetivos.

Es por ello que una buena gerencia será capaz de movilizar todos los recursos necesarios para transformar un resultado de I y $D$-conocimiento- en un resultado útil comercialmente. Puede también imponer la visión y estrategias de la compañía e implicar a todos las funciones, incluida la de I y $\mathrm{D}$, en su puesta práctica con éxito.

Para Guédez (1995), gerenciar es tomar una idea, unas instituciones, una iniciativa, unos sentimientos y convertirlos en propósitos, luego de aplicarles una serie de recursos y de administrarlos a través de determinadas acciones organizacionales. Es también favorecer la tradición de esos propósitos en bienes, servicios o conocimientos.

Las funciones gerenciales básicas se pueden resumir en cinco actividades que son, según Bermúdez (1994) las siguientes:

1. Planificación: formada por todas las actividades gerenciales relacionadas con la preparación para el futuro, las tareas específicas, la cual incluye predicción, fijación de objetivos, diseño de estrategias, desarrollo de políticas y fijación de metas.

2. Organización: incluye las actividades gerenciales que producen una estructura de relaciones.
3. Motivación: abarca esfuerzos dirigidos a moldear el comportamiento humano, indagar el liderazgo, comunicación de equipos de trabajo, gratificación y cambio.

4. Selección de personal: se centra en la administración de personal, salarios, sueldos, prestaciones, políticas, quejas y relaciones públicas.

5. Control: aquellas actividades cuyo objetivo sea asegurar que los resultados reales, se estén logrando a través del control de calidad, financiero, recompensas y sanciones, entre otras.

Padilla (1990), señala a la gerencia como una herramienta básica requerida para mejorar la productividad de las unidades de investigación y desarrollo. Estas pueden ser: gerencia participativa, gerencia científica, gerencia de relaciones humanas, gerencia productiva y gerencia Laissez Faire.

Para este autor, las organizaciones dedicadas a la realización de actividades de investigación y desarrollo son particularmente sensibles al estilo y filosofía gerencial que se aplique. Generalmente, el nivel de desarrollo personal de los miembros de la organización es elevado, por lo que una insistencia exagerada en la productividad conducirá rápidamente a una situación de desgaste de las relaciones humanas y pérdida de la confianza en la gerencia.

Para Avalos (1994), la gerencia de I y $\mathrm{D}$ concierne al conjunto de decisiones relacionadas con las actividades de generación, divulgación y aplicación de conocimientos nuevos y útiles desde el punto de vista productivo. Por su propia naturaleza, estas actividades impli- 
can un alto grado de incertidumbre y de riesgo.

Por otro lado Gordon (1997), plantea que la gerencia de I y $D$ debe fundamentarse en criterios y métodos concebidos y que permitan por consiguiente: planificar actividades que conlleven una alta dosis de aleatoriedad, realizar inversiones en proyectos para lo que resulta complicado calcular la tasa interna de retorno, seleccionar y manejar un personal que trabaja fundamentalmente con su creatividad, conciliar los propósitos de la organización con la libertad de investigación, armonizar las posibilidades técnicas con las características del mercado, y comercializar innovaciones.

Villegas (1991), enumera una serie de habilidades que debe poseer un gerente de I y $D$, entre los cuales menciona: ser líder, organizador y estratega, comunicar los objetivos de la organización y actuar como vocero, habilidades para lidiar con conflictos y resolver problemas, capacidad de formar y mantener un equipo unido, habilidades para motivar, capacidad de construir un clima favorable para las personas, habilidades gerenciales: organización, planeamiento, delegación y toma de decisiones, poseer una formación profesional básica en campo definido del conocimiento.

La gerencia debe adaptarse a los cambios que ocurren en el contexto en que se opera, asumir riesgos responsablemente, y enfrentarse a dichos cambios, de tal modo que la vida de la organización sea perdurable. Debe evidenciar un papel emprendedor.

\section{Resultados de la investigación:}

\subsection{Análisis de los resultados del cuestionario aplicado a los directores ejecutivo de los parques tecnológicos $(n=3)$}

El análisis es realizado de acuerdo a la relación de la operacionalización de variable objeto de estudio, las dimensiones e indicadores y sus respectivos ítems, cuyos resultados son mostrados en tablas sinópticas que representan las frecuencias absolutas que corresponden a cifras numéricas totales de población y en frecuencias relativas que corresponden a valores expresados en porcentajes.

Variable: Estrategia para la Gerencia de I+D

Dimensión: Situación Actual

La dimensión situación actual se estudio, a través de los indicadores: elementos internos y elementos externos.

Indicador: Elementos Internos.

El indicador elementos internos está conformado por todos los aspectos que son considerados como fortalezas y debilidades internas dentro de la organización. El análisis del indicador comienza por la Tabla 1, la cual proporciona información sobre actividades de gerencia general, mercadotecnia, finanzas, producción y operaciones, investigación y desarrollo.

En la Tabla $1(n=3)$ se analizan las alternativas dadas a los Directores Ejecutivos en relación a los aspectos internos en los Parques Tecnológicos.

Se observa que la estructura organizativa, disponibilidad de personal técni- 


\section{Tabla 1 \\ Aspectos internos (fortalezas y debilidades) que están presentes en los parques tecnológicos $n=3$}

\begin{tabular}{|c|c|c|c|c|c|}
\hline & \multicolumn{2}{|c|}{ sí } & \multicolumn{2}{|c|}{ NO } & \multirow[t]{2}{*}{ Total } \\
\hline & $\mathbf{F}$ & $\%$ & $\mathbf{F}$ & $\%$ & \\
\hline Estructura organizativa. & 3 & 100 & & & 100 \\
\hline Disponibilidad del personal técnico gerencial. & 3 & 100 & & & 100 \\
\hline $\begin{array}{l}\text { Presencia del personal técnico calificado para el desem- } \\
\text { peño de funciones de asesoría, consultaría para el sec- } \\
\text { tor industrial. }\end{array}$ & 3 & 100 & & & 100 \\
\hline Apoyo económico & 1 & 33,33 & 2 & 66,66 & 100 \\
\hline $\begin{array}{l}\text { Espacio físico para las instalaciones de empresas de } \\
\text { base tecnológica. }\end{array}$ & 1 & 33,33 & 2 & 66,66 & 100 \\
\hline $\begin{array}{l}\text { Marcada disminución de trámites burocráticos adminis- } \\
\text { trativos con la consecuente agilización de los procesos } \\
\text { de gestión. }\end{array}$ & 2 & 66,66 & 1 & 33,33 & 100 \\
\hline Patentes obtenidas. & 1 & 33,33 & 2 & 66,66 & 100 \\
\hline Mecanismos permanentes de promoción y publicidad. & & & 3 & 100 & 100 \\
\hline $\begin{array}{l}\text { Experiencia en el manejo de modalidades eficientes de } \\
\text { vinculación - empresa. }\end{array}$ & 2 & 66,66 & 1 & 33,33 & 100 \\
\hline $\begin{array}{l}\text { Capacidad de negociación en el área tecnológica con su } \\
\text { potencial mercado en el entorno productivo. }\end{array}$ & 1 & 33,33 & 2 & 66,66 & 100 \\
\hline Planes explícitos de mercadeo tecnológico. & 1 & 33,33 & 2 & 66,66 & 100 \\
\hline $\begin{array}{l}\text { Personal de planta especializado en estrategia de co- } \\
\text { mercialización de tecnología. }\end{array}$ & 1 & 33,33 & 2 & 66,66 & 100 \\
\hline
\end{tabular}

Fuente: Chirinos y Rincón (2006).

co gerencial y presencia de personal técnico calificado para el desempeño de funciones de asesorías se presentan como fortalezas, mientras que como limitantes (debilidades) se menciona los mecanismos permanentes de promoción y publicidad que aparecen con un porcentaje del cien por ciento (100\%). Posteriormente se mencionan capacidad de negociación en el área tecnológica, planes explícitos de mercadeo, personal de planta especializado con un porcentaje del $66,66 \%$.
Continuando con el análisis del indicador elementos internos se presenta la Tabla II, donde se solicita a la población estudiada señale los aspectos que considere más eficiente o no eficiente en los Parques Tecnológicos.

Se observa en la Tabla 2 que la totalidad de los directores coinciden en señalar las alternativas capacidad gerencial, estrategia de negocios, calidad de las respuestas y trámites administrativos como los más eficientes, mientras que el 


\section{Tabla 2 \\ Aspectos considerados como eficientes y no eficientes en los parques tecnológicos $n=3$}

\begin{tabular}{|c|c|c|c|c|c|}
\hline & \multicolumn{2}{|c|}{ sí } & \multicolumn{2}{|c|}{ NO } & \multirow[t]{2}{*}{ Tota } \\
\hline & $\mathbf{F}$ & $\%$ & $\mathbf{F}$ & $\%$ & \\
\hline Capacidad gerencial. & 3 & 100 & & & 100 \\
\hline Estrategias de negocios. & 3 & 100 & & & 100 \\
\hline Integración con el sector industrial. & & & 3 & 100 & 100 \\
\hline Capacidad de respuesta a tiempo. & 2 & 66,66 & 1 & 33,33 & 100 \\
\hline Promoción y publicidad. & & & 3 & 100 & 100 \\
\hline Mercadeo. & 1 & 33,33 & 2 & 66,66 & 100 \\
\hline Calidad de las repuestas. & 3 & 100 & & & 100 \\
\hline Trámites administrativos. & 3 & 100 & & & 100 \\
\hline
\end{tabular}

Fuente: Chirinos y Rincón (2006).

aspecto integración con el sector industrial y promoción y publicidad con el $100 \%$, lo consideran como el más deficiente.

Indicador: Elementos Externos.

El indicador elementos externos está conformado por términos que se refieren a tendencias y hechos económicos, sociales, culturales, ambientales, políticas, jurídicas, gubernamentales, tecnológicos y competitivos que podrían beneficiar o perjudicar significativamente a la organización en el futuro. En la Tabla 3 se recaba información de una serie de aspectos externos (oportunidades) que se consideran están presentes en los parques tecnológicos.

Cuando se solicita a los directores, señalar las oportunidades de los Parques Tecnológicos; el apoyo institucional y financiero por parte de FONACYT aparece en primer lugar (100\%), en segundo lugar, mencionan las alternativas amplio mercado cautivo que representan la pequeña y mediana empresa y amplitud de canales de comunicación y vinculación con la sociedad civil con un $66,66 \%$, mientras que la inexistencia de las organizaciones similares de la región, con las que reducen los ámbitos de competencias se presenta en un aspecto negativo con el $100 \%$. Continuando con el análisis del indicador elementos externos se presenta la Tabla 4, donde se les solicita a los Directores, señalar aquellos factores que constituyen mayor conflicto para los parques tecnológicos.

Los resultados que se analizan en la Tabla 4 están relacionados con los aspectos de mayor conflicto en los Parques Tecnológicos. En este sentido, los directores señalan la alternativa la actual normativa universitaria que en circunstancia inciden provocando retardo de gestión administrativa con el $66,66 \%$. 


\section{Tabla 3 \\ Aspectos externos (oportunidades) que están presentes en los parques tecnológicos $\mathbf{n}=3$}

\begin{tabular}{|c|c|c|c|c|c|}
\hline & \multicolumn{2}{|c|}{ sí } & \multicolumn{2}{|c|}{ NO } & \multirow[t]{2}{*}{ Total } \\
\hline & $\mathbf{F}$ & $\%$ & $\mathbf{F}$ & $\%$ & \\
\hline $\begin{array}{l}\text { Apoyo institucional y financiero que represen- } \\
\text { tan instituciones conexas como FONACYT. }\end{array}$ & 3 & 100 & & & 100 \\
\hline $\begin{array}{l}\text { Inexistencia de organizaciones similares en la } \\
\text { región con lo que reducen los ámbitos de com- } \\
\text { petencia. }\end{array}$ & & & 3 & 100 & 100 \\
\hline $\begin{array}{l}\text { La imagen institucional que representa el entor- } \\
\text { no de la Universidad tanto en el área científica } \\
\text { como en el área de extensión. }\end{array}$ & 1 & 33,33 & 2 & 66,66 & 100 \\
\hline $\begin{array}{l}\text { El amplio mercado cautivo que representa la } \\
\text { pequeña y mediana empresa. }\end{array}$ & 2 & 66,66 & 1 & 33,33 & 100 \\
\hline $\begin{array}{l}\text { Amplitud de canales de comunicación y vincu- } \\
\text { lación con la sociedad civil. }\end{array}$ & 2 & 66,66 & 1 & 33,33 & 100 \\
\hline
\end{tabular}

Fuente: Chirinos y Rincón (2006).

Tabla 4

Factores externos que constituyen mayor conflicto para los parques tecnológicos $n=3$

\begin{tabular}{|c|c|c|c|c|c|c|c|}
\hline & \multicolumn{2}{|c|}{ sí } & \multicolumn{2}{|c|}{ NO } & \multicolumn{2}{|c|}{ N C } & \multirow[t]{2}{*}{ Total } \\
\hline & $\mathbf{F}$ & $\%$ & $\mathbf{F}$ & $\%$ & $\mathbf{F}$ & $\%$ & \\
\hline $\begin{array}{l}\text { La actual normativa universitaria que en cir- } \\
\text { cunstancia inciden provocando retardo de ges- } \\
\text { tión administrativa. }\end{array}$ & 2 & 66,66 & & & 1 & 33,33 & 100 \\
\hline $\begin{array}{l}\text { La escasa voluntad de la banca comercial pri- } \\
\text { vada a coparticipar o financiar actividades de } \\
\text { innovación tecnológica. }\end{array}$ & 1 & 33,33 & 1 & 33,33 & 1 & 33,33 & 100 \\
\hline $\begin{array}{l}\text { La presencia de otras dependencias universi- } \\
\text { tarias tratando de incursionar los ámbitos de } \\
\text { competencia del parque tecnológico. }\end{array}$ & 1 & 33,33 & 1 & 33,33 & 1 & 33,33 & 100 \\
\hline $\begin{array}{l}\text { Los altos costos de adquisición de insumo - } \\
\text { equipos de investigación y materiales biblio- } \\
\text { gráficos. }\end{array}$ & 1 & 33,33 & 1 & 33,33 & 1 & 33,33 & 100 \\
\hline $\begin{array}{l}\text { La natural resistencia del empresariado regio- } \\
\text { nal y nacional de establecer relaciones comer- } \\
\text { ciales con dependencia universitaria. }\end{array}$ & 1 & 33,33 & 1 & 33,33 & 1 & 33,33 & 100 \\
\hline
\end{tabular}

Fuente: Chirinos y Rincón (2006). 
3.2 Análisis de los resultados del cuestionario $\mathrm{B}$, aplicado a los investigadores adscritos a los parques tecnológicos $(n=13)$ cia de I+D

Variable: Estrategia para la Geren-

Dimensión: Situación Actual

La dimensión situación actual se estudia integrando a los indicadores elementos internos y elementos externos.

Indicador: Elementos Internos

El indicador elementos internos está conformado por todos los aspectos que son considerados como fuerzas y debilidades internas que son características que controlan la organización y que desempeña muy bien o muy mal. El análisis de este indicador se comienza por la Tabla 5 , la cual recaba información sobre los aspectos internos (Fortalezas y Debilidades) presentes en los parques tecnológicos.

La Tabla 5 refleja los resultados de los aspectos internos que están presentes en los parques tecnológicos, los investigadores señalan las alternativas, ambientes, organizacional favorable para

\section{Tabla 5}

\section{Aspectos internos presentes en los parques tecnológicos} (fortalezas y debilidades) $n=13$

\begin{tabular}{|c|c|c|c|c|c|c|c|}
\hline & \multicolumn{2}{|c|}{ Sí } & \multicolumn{2}{|c|}{ NO } & \multicolumn{2}{|c|}{ N C } & \multirow[t]{2}{*}{ Total } \\
\hline & $\mathbf{F}$ & $\%$ & $F$ & $\%$ & $\mathbf{F}$ & $\%$ & \\
\hline $\begin{array}{l}\text { Espacio físico suficiente y acorde para } \\
\text { realizar investigación. }\end{array}$ & 4 & 30,76 & 9 & 69,23 & & & 100 \\
\hline $\begin{array}{l}\text { Ambiente organizacional favorable para } \\
\text { la investigación. }\end{array}$ & 11 & 84,61 & 2 & 15,38 & & & 100 \\
\hline $\begin{array}{l}\text { Apoyo económico y de gestión institucio- } \\
\text { nal. }\end{array}$ & 2 & 15,38 & 11 & 84,61 & & & 100 \\
\hline $\begin{array}{l}\text { Marcada disminución de tramite burocrá- } \\
\text { tico administrativo. }\end{array}$ & 7 & 53,84 & 6 & 46,15 & & & 100 \\
\hline $\begin{array}{l}\text { Seguimiento y evaluación de las activi- } \\
\text { dades de investigación. }\end{array}$ & 4 & 30,76 & 9 & 69,23 & & & 100 \\
\hline $\begin{array}{l}\text { Infraestructura para poder cumplir con } \\
\text { las actividades de I+D. }\end{array}$ & 5 & 38,46 & 8 & 61,53 & & & 100 \\
\hline $\begin{array}{l}\text { Presupuesto asignado a los proyectos } \\
\text { de investigación. }\end{array}$ & 1 & 7,69 & 12 & 92,30 & & & 100 \\
\hline $\begin{array}{l}\text { La burocracia administrativa que deben } \\
\text { seguir los proyectos de investigación. }\end{array}$ & 5 & 38,46 & 6 & 46,15 & 2 & 15,38 & 100 \\
\hline $\begin{array}{l}\text { Claridad en las normas que rigen la in- } \\
\text { vestigación. }\end{array}$ & 6 & 46,15 & 7 & 53,84 & & & 100 \\
\hline $\begin{array}{l}\text { Vinculación investigador - universidad - } \\
\text { entorno. }\end{array}$ & 6 & 46,15 & 7 & 53,84 & & & 100 \\
\hline
\end{tabular}

Fuente: Chirinos y Rincón (2006). SI: Fortalezas. NO: Debilidades. NC: No Contesto. 
la investigación con un porcentaje del 84,61\%; marcada disminución de transmite burocrático administrativo le dan un porcentaje del $53,84 \%$. En cambio como debilidades señalan el presupuesto asignado a los proyectos de investigación con un $92,30 \%$, apoyo económico y de gestión institucional con un $84,61 \%$, seguimiento y evaluación de las actividades y espacio físico suficiente y acorde para realizar investigación (69,23\%), e infraestructura para poder cumplir con las actividades de investigación (61,53\%).

Siguiendo con el análisis de los resultados del indicador de elementos internos se ofrece la Tabla 6 , donde se solicita a la población encuestada, señalar los factores que inciden en el nivel de desempeño de la organización.

En cuanto al nivel de desempeño de la organización donde laboran los investigadores, ellos señalan la calidad de las instalaciones y equipos con un 92,30\%; disponibilidad de los recursos humanos con un $84,61 \%$, armonía de las relaciones interpersonales y disponibilidad de los servicios de información con un $69,23 \%$.

Indicador: Elementos Externos

El indicador elementos externos está conformado por todos los aspectos que son considerados como amenazas y oportunidades que están, en gran medida fuera del control de una organización cualquiera, de ahí el término "externas".

En el análisis de los items del indicador elementos externos se indaga información sobre los aspectos externos presentes en los parques tecnológicos (Tabla 7).

Entre los aspectos que se consideran como oportunidades presentes en los Parques Tecnológicos se señala el apoyo institucional y financiero por parte FONACYT, amplio mercado cautivo que representa la pequeña y mediana empresa con el $84,61 \%$; luego, la imagen institucional que representa el entorno universitario con el $76,92 \%$ y amplitud de canales de comunicación y vinculación con la sociedad civil con el $69,23 \%$.

\section{Tabla 6}

Factores que inciden en el nivel de desempeño de la organización $\mathbf{n}=13$

\begin{tabular}{lccccccc}
\hline & \multicolumn{3}{c}{ Sí } & \multicolumn{3}{c}{ NO } & \multicolumn{2}{c}{ N C } & \multirow{2}{*}{ Total } \\
\cline { 2 - 6 } & F & $\%$ & F & $\%$ & F & $\%$ & \\
\hline Calidad de las instalaciones y equipos. & 12 & 92,30 & 1 & 7,69 & & 100 \\
Liderazgo de la dirección de unidad. & 8 & 61,53 & 5 & 38,46 & & 100 \\
Disponibilidad de los recursos humanos. & 11 & 84,61 & 2 & 15,38 & & 100 \\
Prestigio actual de los investigadores. & 6 & 46,15 & 7 & 53,84 & & 100 \\
Armonía de las relaciones interpersonales. & 9 & 69,23 & 4 & 30,76 & & 100 \\
Contacto con otros centros I+D. & 6 & 46,15 & 6 & 46,15 & 1 & 7,69 & 100 \\
Disponibilidad de los servicios de información. & 9 & 69,23 & 4 & 30,76 & & 100 \\
Adecuada organización. & 7 & 53,84 & 6 & 46,15 & & 100 \\
\hline
\end{tabular}

Fuente: Chirinos y Rincón (2006) SI: Inciden en el nivel de desempeño. NO: No inciden. NC: No contestó. 


\section{Tabla 7 \\ Aspectos externos presentes en los parques tecnológicos (oportunidades y amenazas) $n=13$}

\begin{tabular}{|c|c|c|c|c|c|c|c|}
\hline & \multicolumn{2}{|c|}{ Sí } & \multicolumn{2}{|c|}{ NO } & \multicolumn{2}{|c|}{ N C } & \multirow[t]{2}{*}{ Total } \\
\hline & $\mathbf{F}$ & $\%$ & $\mathbf{F}$ & $\%$ & $\mathbf{F}$ & $\%$ & \\
\hline $\begin{array}{l}\text { Apoyo institucional y financiero por parte } \\
\text { de FONACYT. }\end{array}$ & 11 & 84,61 & 2 & 15,38 & & & 100 \\
\hline $\begin{array}{l}\text { La imagen institucional que representa el } \\
\text { entorno universitario (área científica y } \\
\text { extensión). }\end{array}$ & 10 & 76,92 & 2 & 15,38 & 1 & 7,69 & 100 \\
\hline $\begin{array}{l}\text { Amplitud de canales de comunicación y } \\
\text { vinculación con la sociedad civil. }\end{array}$ & 9 & 69,23 & 4 & 30,76 & & & 100 \\
\hline $\begin{array}{l}\text { El amplio mercado cautivo que represen- } \\
\text { ta la pequeña y mediana empresa. }\end{array}$ & 11 & 84,61 & 2 & 15,38 & & & 100 \\
\hline $\begin{array}{l}\text { La actual normativa universitaria que } \\
\text { provoca retardos administrativos. }\end{array}$ & 3 & 23,07 & 10 & 76,92 & & & 100 \\
\hline $\begin{array}{l}\text { La poca voluntad de la banca comercial } \\
\text { privada a financiar actividades de inno- } \\
\text { vación. }\end{array}$ & 1 & 7,69 & 12 & 92,30 & & & 100 \\
\hline $\begin{array}{l}\text { Los altos costo de insumo / equipos de } \\
\text { investigación y apoyo bibliográfico. }\end{array}$ & 2 & 15,38 & 11 & 84,61 & & & 100 \\
\hline $\begin{array}{l}\text { La resistencia del empresariado a esta- } \\
\text { blecer relaciones comerciales con la de- } \\
\text { pendencia universitaria. }\end{array}$ & 1 & 7,69 & 10 & 76,92 & 2 & 15,38 & 100 \\
\hline
\end{tabular}

Fuente: Chirinos y Rincón (2006) SI: Oportunidades. NO: Amenazas NC: NO contestó.

No obstante, dentro de los aspectos limitantes (amenazas) se indica la poca voluntad de la Banca Comercial Privada a financiar actividades de innovación $(92,30 \%)$, los altos costos de insumos-equipos de investigación y apoyo bibliográfico con el $84,61 \%$, la actual normativa universitaria que provoca retardos administrativos $76,92 \%$ y la resistencia del empresariado a establecer relaciones comerciales con la dependencia universitaria $76,92 \%$.

\section{A manera de conclusión}

Los resultados del análisis estratégico de la Gerencia de Investigación y Desarrollo de los Parques Tecnológicos, permite establecer que las fortalezas y debilidades internas, así como, las oportunidades y amenazas se encuentran presentes en los parques tecnológicos.

Al analizar los resultados de la pertinencia en cuanto al posionamiento interno permite derivar que existen fortalezas como estructura organizativa, disponibili- 
dad de personal técnico gerencial, presencia de personal técnico calificado, ambiente organizacional favorable para la investigación. No obstante se destacan como aspectos limitantes (debilidades) mecanismos permanentes de promoción y publicidad, apoyo económico y de gestión institucional, presupuesto asignado a los proyectos de investigación, espacio físico suficiente y acorde para realizar investigación, seguimiento y evaluación de las actividades de investigación, infraestructura para poder cumplir con las actividades de I + D, patentes obtenidos, capacidad de negociación en el área tecnológica con su potencial mercado en el entorno productivo, planes explícitos de mercadeo tecnológico y personal de planta especializado en estrategia de comercialización de tecnología.

El proceso de identificar y evaluar las fuerzas y las debilidades de la organización en las áreas funcionales de un negocio es una actitud vital de la administración estratégica.

En relación a los aspectos considerados como eficientes y no eficientes los resultados coinciden en que existe capacidad gerencial, estrategias de negocios, calidad de respuesta a tiempo, y trámites administrativos mientras que consideran como los más deficientes la integración con el sector industrial, la promoción, publicidad y el mercadeo.

Los resultados referidos a los elementos internos en el cual se estudian los factores que inciden en el nivel de desempeño de la organización, se puede afirmar que los investigadores indican como importantes la calidad de las instalaciones y equipos, la disponibilidad de los recursos humanos, la armonía de las rela- ciones interpersonales y la disponibilidad de los servicios de información.

En el análisis del posicionamiento externo los investigadores señalan como oportunidades el apoyo institucional y financiero que representan instituciones conexas como FONACYT, la imagen institucional que representan el entorno universitario (área científica y extensión), el amplio mercado cautivo que representa la pequeña y mediana empresa y amplitud de canales de comunicación y vinculación con la sociedad civil. Dentro del mismo análisis se observa como amenazas la poca voluntad de la banca comercial privada a financiar actividades de innovación, los altos costos de insumos-equipos de investigación y apoyo bibliográfico, la actual normativa universitaria que provoca retardos administrativos y la resistencia del empresariado a establecer relaciones comerciales con la dependencia universitaria.

Las amenazas y oportunidades están en gran medida fuera del control de una organización cualquiera, de allí el término "externas". El conocimiento de las oportunidades, tanto en el ambiente externo como dentro de la organización, es el punto de partida real para la planeación. Es importante observar previamente todas las posibles oportunidades futuras y verlas con claridad y por completo.

En síntesis, los resultados presentados a partir del análisis estratégico realizado en los Parques Tecnológicos del occidente del País indican: la pertinencia en cuanto al posicionamiento interno permite derivar que existen fortalezas como estructura organizativa, disponibilidad del personal técnico gerencial, presencia del personal técnico calificado, ambiente organizacional favorable para la investi- 
gación, sin embargo, se observan debilidades, como pocos mecanismos permanentes de formación y publicidad, insuficiente apoyo económico y de gestión institucional, escaso presupuesto asignado a los proyectos de investigación, espacio físico insuficiente para realizar investigación, poco seguimiento y evaluación de las actividades de investigación, pocas patentes obtenidas, insuficiente capacidad de negociación en el área tecnológica, inexistencia de planes explícitos de mercadeo tecnológico y escaso personal de planta especializado en estrategia de comercialización de tecnología.

Entre los aspectos considerados como eficientes se determina la capacidad gerencial, estratégicas de negocios, calidad de las respuestas y trámites administrativos, sin embargo, entre los más deficientes se presentan la integración con el sector industrial y la promoción y publicidad.

En el análisis del posicionamiento externo se determina que existen oportunidades como el apoyo institucional que representan instituciones conexas como FONACYT, la imagen institucional que representa el entorno, el amplio mercado continuo que representa la pequeña y mediana empresas y amplitud de canales de comunicación y vinculación con al sociedad civil.

Dentro del mismo análisis se observan como amenazas la poca voluntad de la banca comercial privada a financiar actividades de innovación, los altos costos de insumos-equipos de investigación y apoyo bibliográfico, la actual normativa universitaria que provoca retardos administrativos y la resistencia del empresariado a establecer relaciones comerciales con la dependencia universitaria.

\section{Referencias Bibliográficas}

Avalos, Ignacio (1994). Aproximación a la gerencia de tecnología en la empresa. Ediciones IESA. Caracas.

Baena, Dolores (2003). La medición de la investigación científica y el desarrollo tecnológico (I + D). Principales indicadores. Rev. Electrónica de Geografía y Ciencias Sociales. Barcelona España. 69 (34).

Bermúdez, O. (1994). La planificación y gerencia de un centro de investigación y desarrollo en el área frutícola: El Centro Frutícola del Estado Zulia. Tesis de Maestría. LUZ. Maracaibo.

David, Fred (1990). La gerencia estratégica. Legis Editores. Tercera Edición. Bogotá-Colombia.

David, Fred (1997). Conceptos de administración estratégica. Quinta Edición. Legis Editores. Bogotá-Colombia. 355. pp.- 1997.

Figueroa, Marta (1996). Propuesta de Gerencia para un instituto de investigación y desarrollo. Estudio de Caso: Instituto de Investigaciones Petroleras de LUZ. Tesis de Maestría.

Gordon, Juan (1997). Comportamiento organizacional. México: A. Simón y Schuster Company.

Guedez, Víctor (1995). Gerencia, cultura y educación. Editorial Tropykos / Clacdec. Caracas, Venezuela.

Koontz, Harold y Weihrich, H. (2001). Elementos de administración. McGraw Hill Interamericana. Quinta Edición. México. 497 pp.

Padilla, Rafael (1990). Modelo de gerencia en investigación y desarrollo. Revista Espacio Vol. 11. № 1. Caracas.

Parisca, Simón (1992). Sistema venezolano de innovación tecnológica. Rev 13: (3) 21-40. 
Seaton, Carlos (2000). "El mercadeo relacional y de servicios tecnológicos. Las asignaturas pendientes de la mayoría de las organizaciones públicas de investigación y desarrollo". Revista Espacios 21: (2) 1-5.

Stoner, James; Freeman, E. y Gilbert, Daniel (1996). Administración. Editorial Prentice Hall Hispanoamericana, S.A. Sexta Edición. México. 691pp.
Thompson A. y Strickland (1998). Dirección y administración estratégica. Mc Graw Hill. México.

Villegas, José Manuel (1991). Desarrollo Gerencial. Enfoque conceptual y metodológico. Ediciones Vega. Caracas.

Viana Horacio, Cervilla Miguel, Avalos Ignacio, Balaguer Antonio (1994). "La capacidad y la competitividad de la industria manufacturera venezolana". Revista Espacios № 15. Vol. 1. 